\title{
Improvement prostate cancer detection rate of suspicious lesions through MRI/TRUS fusion guided biopsy by a multiteam of radiologists
}

\author{
Nicolò Fiorello ${ }^{1 *}\left(\mathbb{D}\right.$, Andrea Mogorovich ${ }^{1}$, Andrea Di Benedetto ${ }^{1}$, Daniele Summonti ${ }^{1}$, Carlo Tessa ${ }^{2}$, \\ Laura Turturici ${ }^{2}$, Andrea Mannini' ${ }^{2}$, Michele Bellucci ${ }^{2}$, Claudio Vignali² and Carlo Alberto Sepich
}

\begin{abstract}
Background: The objective of our study was to analyze the data of our biopsies, determine a detection rate (DR), compare it with the data in the literature and draw possible deductions, so as to offer the patient the possibility of not having other biopsies in the future.

Methods: We have enrolled 189 biopsy-naive patients in the period between September 2018 and December 2020. Each patient underwent multiparametric (mp)-MRI which was reviewed by our team of radiologists. In our center, each examination is examined by 4 radiologists separately with an overall final result. Through the $t$ student test, any statistically significant differences between the DRs and the concordance rate between the positive cores and the suspected area on MRI were analyzed for each urologist who performed the procedure.

Results: The absolute (DR) was 69.3\% (131/189 patients). The relative DR for each PIRADS score was 41\% for PIRADS 3,70.2\% for PIRADS 4, 89.3\% for PIRADS 5. We found a high percentage of agreement between the positive biopsy samples and the suspicious area identified on MRI: $90.8 \%$ (119/131 patients). There were no statistically significant differences between the DRs of the urologists who performed the procedure $(p=0.89)$, nor for the percentage of agreement between the positivity of the core and the suspected area on MRI $(p=0.92)$.

Conclusions: MRI in the future could become the gold standard for performing MRI fusion-guided biopsies to have a better diagnostic result and avoid rebiopsies. A team MRI reading allows greater accuracy in identifying the suspected lesion, which is demonstrated by a high rate of agreement with the positivity of the cores $(90.8 \%)$. There is a cost problem due to the need to carry out the mpMRI but it could have less impact in the future. In addition, the MRI provides useful information on the extent of the disease (e.g., cT3a/b) which allows you to better plan the surgical strategy or other therapies.
\end{abstract}

Keywords: Imaging in urology, Prostate cancer, Urologic oncology, mp-MRI, Detection rate of prostate cancer, Prostatic fusion biopsy, Software-guided fusion biopsy

*Correspondence: fiorellourologia@gmail.com

${ }^{1}$ UOC Urologia Ospedale Versilia, Camaiore, LU, Italy

Full list of author information is available at the end of the article

\section{Background}

Prostate cancer (PC) is the second most commonly diagnosed cancer in men, with an estimated 1.1 million diagnoses worldwide in 2012, accounting for $15 \%$ of all cancers diagnosed [1]. PC is the fourth most common cancer type in Egypt with an incidence rate of about $4.5 \%$ among male cancer patients. Despite having 
1 Very Low: Clinically significant disease is highly unlikely to be present. 2 Low: Clinically significant disease is unlikely to be present. 3 Intermediate: Clinically significant disease is equivocal. 4 High: Clinically significant disease is likely to be present. 5 Very High: Clinically significant disease is highly likely to be present.

Fig. 1 PIRADS $v 2$ assessment categories

a low mortality rate, prostate cancer greatly impacts the patients' quality of life [2].

Multiparametric magnetic resonance imaging (mpMRI) combining T2-weighted (T2w) imaging with diffusion-weighted (Dw) imaging, dynamic contrast-enhanced (DCE) imaging and/or magnetic resonance spectroscopy (MRS) has yielded promising results in prostate cancer detection and localization [2, 3].

T2-weighted sequences of the prostate provide anatomic information and should include a triplanar (axial, coronal, and sagittal) or comparable sequence [4].

The PIRADS v2 has given guidance to standardize zonal reporting, which helps urologists use information from the report regarding on $\mathrm{T} 2$-weighted sequence and zonal location to identify suspicious areas [5].

The current literature regarding the role of multiparametric MRI and fusion-guided biopsies in urologic practice was reviewed establishing improved accuracy of fusion biopsies over systematic biopsies. They are a superior method for detection of the true grade of cancer for both biopsy naive and patients with prior negative biopsies, choosing appropriate candidates for active surveillance, and monitoring progression on active surveillance [6].

The mp-MRI has a good ability to identify significant lesions, which can be confirmed by prostate biopsy or on surgical specimen, although some lesions (given the multifocality of the PC) are not identified by MRI [7]. The increase in mp-MRI is steadily increasing but many countries face the problem of availability and costs. Prostate mpMRI is used by Italian urologists mainly for detection and for preoperative staging of PCa. Further improvements in terms of mpMRI availability and report standardization are required [8].

\section{Method}

An increasing number of MRI-fusion biopsies are performed in our center. After more than two years of experience, we have examined the detection rate (DR) of biopsy-naive patients and looked for possible correlations for a DR of our center higher than many data in the literature (Fig. 1).

In our study, we have enrolled 189 biopsy-naive patients in the period between September 2018 and
December 2020. Each patient underwent mp-MRI which was reviewed by our team of radiologists. In our center, each examination is examined by 4 radiologists separately, with moderate experience in mp-MRI (each has read over $60 \mathrm{mp}-\mathrm{MRI})$, with an overall final result. The evaluation score system was PIRADS score v2. Each radiologist assigned a PIRADS score to be attributed to the suspicious area in his opinion. If there was agreement in the report of the 4 radiologists, no group review was expected. Otherwise, they were reviewed collectively until a final report was created.

The median value of PSA was $8.3 \mathrm{ng} / \mathrm{dL}$ (Range $0.48-$ 50.1). For each biopsy the PIRADS score, the clinical staging (cTNM) was evaluated using the MRI, the PSA value and the histological result. The concordance between the PIRADS area identified and the positive histological sample was also evaluated. All biopsies were performed MRI-targeted+systematic. The procedures were performed by 4 fully trained urologists for standard transrectal ultrasound-guided biopsy (each performed over 100 procedures) which followed a further update to be able to start performing software-based fusion biopsy (Fig. 2).

In our center we only use the transrectal approach.

To image the prostate, the patient will be placed on an MRI table. A series of images will be taken of the prostate and its surrounding area. For the best resolution of the images it is important for the patient to lie very still during the test. A special dye visible on the MRI will be injected intravenously toward the end of the examination. The MRI takes about 45-60 min.

MRI of the prostate was performed using a $1.5 \mathrm{~T}$ MR scanner system (MAGNETOM Avanto, Siemens Healthcare, Erlangen, Germany) equipped with $45 \mathrm{mT} / \mathrm{m}$ gradients strength and a phased array coil together with an endorectal coil. MRI protocol included a T1-weighted TSE sequence in the axial plane (FOV $400 \mathrm{~mm}$, matrix size $236 \times 384 \mathrm{~mm}$, typical TR $583 \mathrm{~ms}$, TE $10 \mathrm{~ms}$, slice thickness $3 \mathrm{~mm}$ ). High-resolution T2-weighted TSE sequences were obtained in the axial, sagittal and coronal planes (FOV $200 \mathrm{~mm}$, matrix size $320 \times 320$, typical TR $3970 \mathrm{~ms}$, TE $120 \mathrm{~ms}$, slice thickness $3 \mathrm{~mm}$, without gap) (Fig. 3).

Diffusion weighted images (DWI; FOV $260 \times 221$, matrix size $97 \times 162$, typical TR $5200 \mathrm{~ms}$, TE $89 \mathrm{~ms}$, $b$-values $50-1000-1400 \mathrm{~s} / \mathrm{mm}^{2}$, slice thickness $3 \mathrm{~mm}$ ) and dynamic contrast enhancement (DCE) images (FOV 200, matrix size $160 \times 160$, TR $4.65 \mathrm{~ms}$, TE $1.64 \mathrm{~ms}$, slice thickness $3 \mathrm{~mm}$ ) were obtained in the axial plane, matching the high-resolution T2-weighted images.

The software used was BkFusion-MIM ${ }^{\circledR}$ 6.9.4 ${ }^{\circledR}$

The radiologist will analyze the images and help to create a three dimensional image of the prostate, 


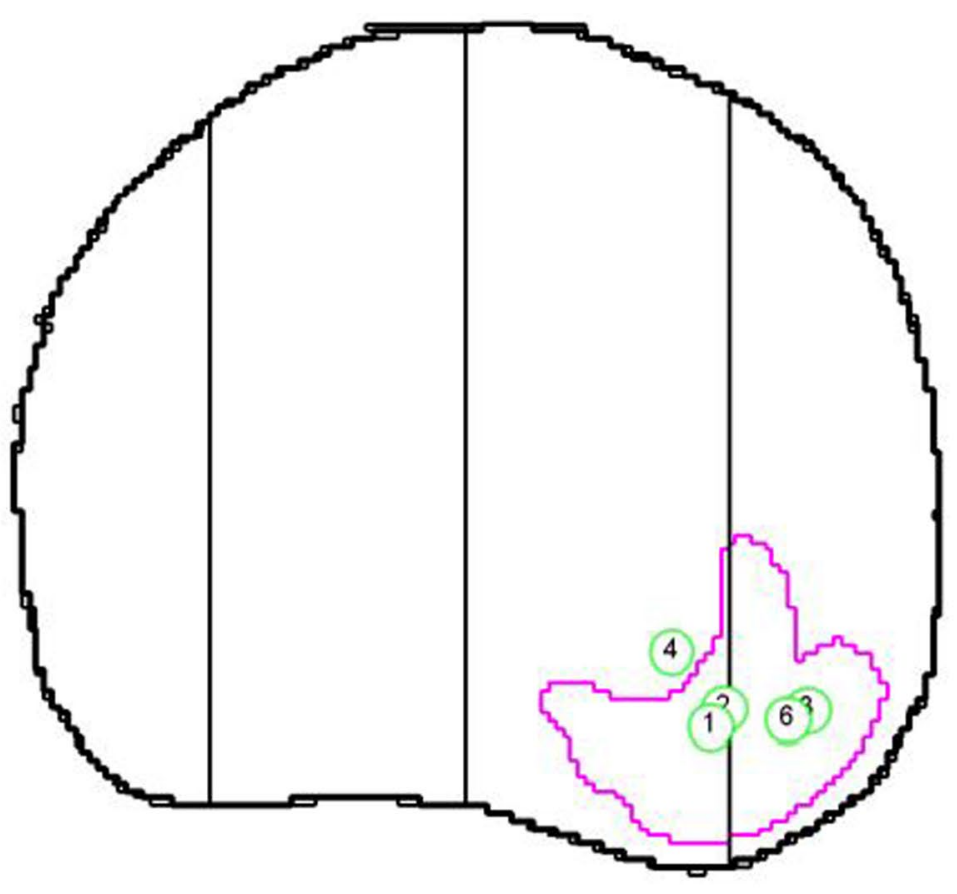

Biopsy Sector View Apex

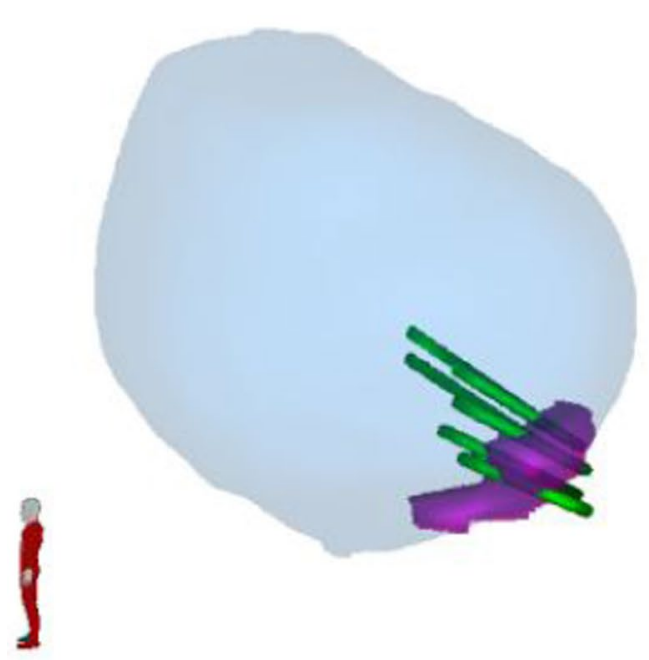

Contour and Dose Surfaces Preview

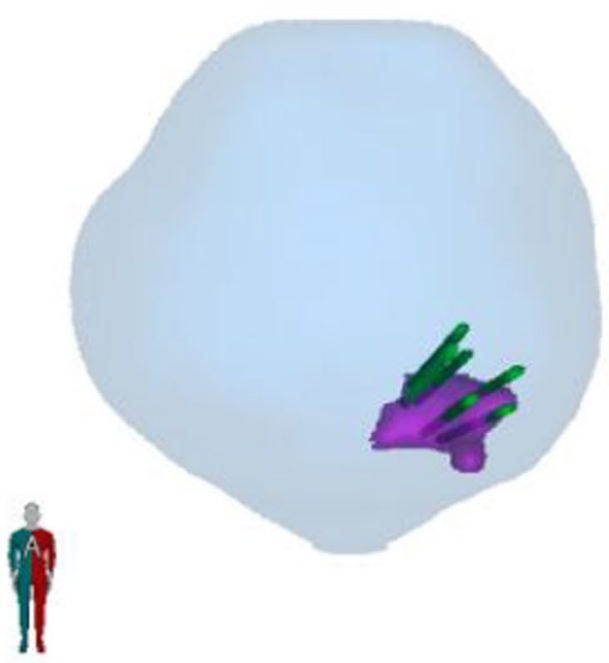

Contour and Dose Surfaces Preview

Fig. 2 a Biopsy Sector View, with the prostatic anatomical diagram; b, c. Contour and Dose Surfaces Preview, with the three-dimensional diagram of perfomed biopsies. The green line shows the trajectory of biopsy inside the suspicious area (bordered by purple line) during transrectal biopsy

indicating abnormal areas or suspicious lesions, marking these on the images. These images will then be transferred electronically to the urologist's procedure room, where the biopsy will take place. Antibiotic prophylaxis is started the day before (usually amoxicillin/clavulanate, fosfomycin or, alternatively fluoroquinolones), and digital rectal examination (DRE) was performed in order to evaluate any potential anatomic or pathologic condition.

Prostate biopsies are performed under transrectal ultrasound guidance. MRI/Ultrasound Fusion technology now allows you to use the obtained MRI images and target suspicious areas that have been identified on those images by the radiologist. Before performing the 


\section{Visualization}
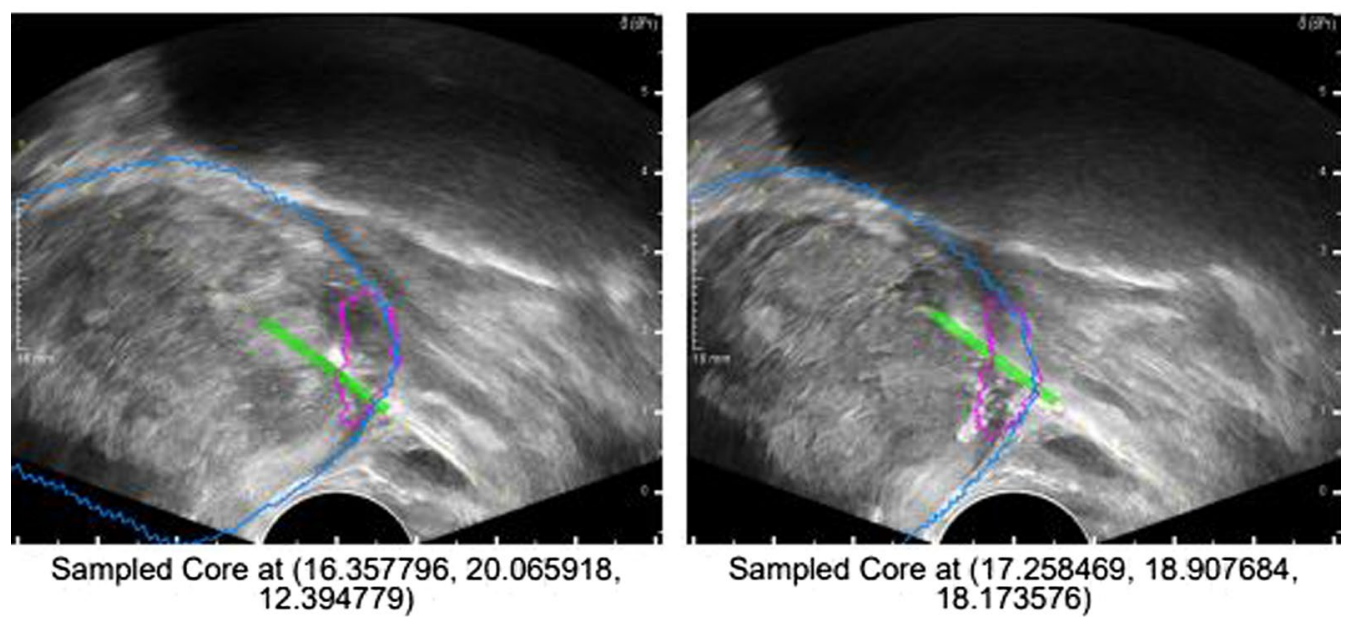

Fig. 3 TRUS view during fusion biopsy. The green line shows the trajectory of biopsy inside the suspicious area

biopsy, the urologist must make the contour to identify the images seen on MRI and make them coincide with the suspicious areas marked on the images available on the ultrasound monitor. These images will then be merged with the real-time transrectal ultrasound image of the prostate. This will allow for targeted sampling of suspicious areas (usually 1-4). Once the target cores have been performed, systematics can be carried out in the remaining parts of the prostate. Normally a random biopsy involves 12 samples in all (6 for each lobe, 1 for each portion of the prostate). Target cores may vary at the discretion of the urologist. So, if, for example, 3 target withdrawals are carried out on the "right lateral base" area, the remaining 11 cores must be added, therefore 14 in total (Fig. 4).

Through the $t$ student test, any statistically significant differences between the DRs and the concordance rate between the positive cores and the suspected area on MRI were analyzed for each urologist who performed the procedure.

\section{Results}

The absolute detection rate (DR) was 69.3\% (129/189 patients). Sixty-eight patients had PIRADS score 3 on MRI, 74 patients PIRADS score 4, 47 patients PIRADS score 5 .

The relative DR for each PIRADS score was $41 \%$ for PIRADS 3, 70,2\% for PIRADS 4, 89.3\% for PIRADS 5.

Clinical staging was assessed on the basis of MRI and histology. Positivity of the samples bilaterally was considered cT2c (according to the EAU guidelines, non-palpable and biopsy positive prostate cancer, with no evidence of extracapsular extension to MRI should be considered
T1c, but is often discordant from the final histological examination).

Thirty-three patients had $3+3$ Gleason Score (GS), 44 patients had $3+4 \mathrm{GS}, 31$ patients had $4+3$ GS, 11 patients had $4+4$ GS, 6 patients had $4+5$ GS, 1 patient had $5+4$ GS and 3 patients had ASAP (Atypical small acinar proliferation).

Patients with GS $3+3$ can be considered clinically insignificant, potentially candidates for active surveillance, according to the number of positive cores or patient choice. Of these, 10 were classified as PIRADS 3 lesions, 16 as PIRADS 4 and 7 as PIRADS 5. Thus it is evident that the PIRADS classification does not directly correlate with the grade of the tumor (Fig. 5).

We found a high percentage of agreement between the positive biopsy samples and the suspicious area identified on MRI: $90.8 \%$ (119/129 patients). The degree of agreement was not affected by the PIRADS score.

Grading of positive biopsies was not always correlated with PIRADS score, although slightly higher in patients with PIRADS 5.

There were no statistically significant differences between the DRs of the urologists who performed the procedure $(p=0.89)$, nor for the percentage of agreement between the positivity of the core and the suspected area on MRI $(p=0.92)$.

\section{Discussion}

Currently the European guidelines do not oblige to perform MRI in naive patients. Nevertheless, emerging clinical trial data support the adoption of this technology as part of the standard of care for the diagnosis of PC [9]. 

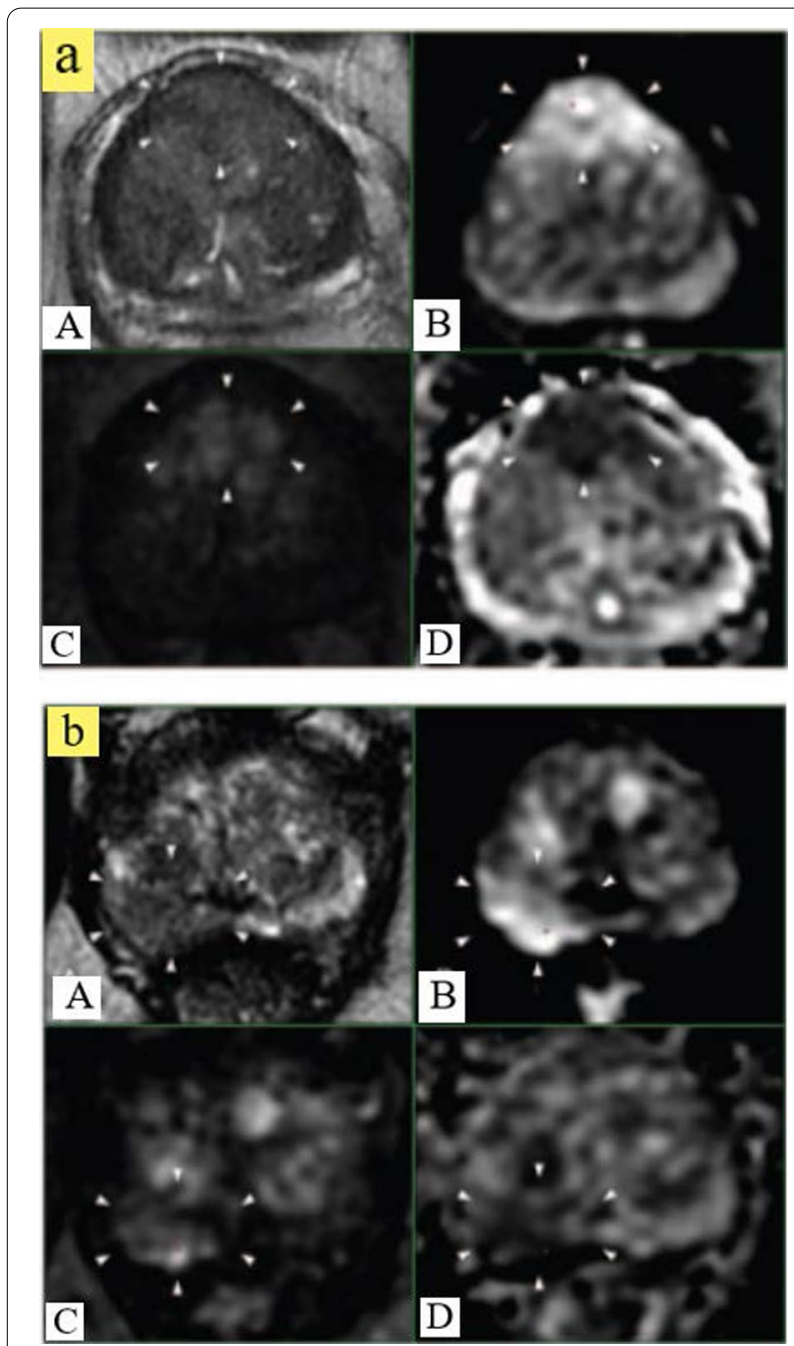

Fig. 4 a Suspicious lesion found in the transitional zone (TZ). MRI examination shows an elliptical focus of homogenous decreased T2 signal ( $A$; axial T2W), increased DWI signal ( $B$; axial DWI b-800), hypervascularity ( $C$; axial DCE-MRI), and reduced signal on ADC ( $D$; axial ADC map), within the anterior transition zone. b Suspicious lesion in peripheral zone (PZ), MRI examination shows an elliptical focus of decreased T2 signal ( $A$; axial T2W), increased DWI signal ( $B_{\text {; }}$ axial DWI b-800), hypervascularity (C; axial DCE-MRI), and reduced signal on ADC (D; axial ADC map), within the right dorsolateral peripheral zone

In a study of Veeru Kasivisvanathan et al. the detection rate of MRI-targeted biopsies and standard biopsies was compared. In this study a total of 500 men underwent randomization. In the MRI-targeted biopsy group, 71 of 252 men (28\%) had MRI results that were not suggestive of prostate cancer, so they did not undergo biopsy. Clinically significant cancer was detected in 95 men (38\%) in the MRI-targeted biopsy group, as compared with 64 of 248 (26\%) in the standard-biopsy group [10].
There are many data in the literature on the DR but they are very varied and it is difficult to establish a limit within which to define the accuracy of MRI fusionguided biopsy [11].

In a study of Tang et al., which totally 13 cohorts in 12 studies, with 3225 men were included, targeted biopsies had a DR of $43.1 \%$ [12].

Analyzing some data in the literature, such as the study of D'Agostino et al. where the DR for PIRADS 3, 4, and 5 were $27.8 \%, 63.6 \%$ and 60 , respectively [12], and comparing it with our data we believe that novelty in our study, which probably allowed us to have a higher DR, was having a multiteam of radiologists who made the MRI more accurate. This allows a better DR with less need of having to undergo the patients to a new biopsy.

We also believe that biopsies must be performed target + systematic to have a complete clinical staging and, although infrequent, be able to have positive biopsies even in areas not suspected by MRI.

Another question is whether to prefer the transrectal biopsy (TRB), more classic and familiar to urologists, or the transperineal biopsy (TPB), recently on the rise with some potential advantages.

The detection rates of the TPB are equivalent or higher than the TRB. In addition, TRB carries a small but clinically significant and increasing risk of sepsis. TPB, whether targeted for MRI or not, consistently results in zero or near zero rates of serious infections. Another important advantage is greater accessibility to front withdrawals. [13].

Despite the urologist's greater familiarity in performing $\mathrm{TRB}$, the future direction, including our center, will likely be to perform TPB only.

There are currently several studies in the literature on the need to use bipatametric (bp) MRI Vs mpMRI. Sometimes bpMRI can give the same results as mpMRI but several procedures are required for such a result (700800 cases as threshold for reliable interpretation with bpMRI) [14]. However, other studies, such as that by Mostafa Alabousi et al., show no significant difference in diagnostic test accuracy was found between mpMRI and bpMRI in the diagnosis of prostate cancer in treatmentnaive patients, with the advantage that bpMRI may represent a faster, cheaper and gadolinium-free alternative to mpMRI $[15,16]$.

It is not possible to predict in retrospect how many biopsies could be positive without the PIRADS system but, since there is high percentage of agreement between the positive biopsy samples and the suspicious area identified on MRI (90.8\%), it is reasonable to think the DR would have been lower. Furthermore, it is difficult to establish whether the same DR would have been obtained using the cognitive fusion technique. However in the 
TO $\rightarrow$ 57/179 (31\%)

Tis $\rightarrow 6 / 179$ (3\%)

$\mathrm{T} 2 \mathrm{a} \rightarrow 50 / 179$ (28\%)

$\mathrm{T} 2 \mathrm{~b} \rightarrow 19 / 179$ (10\%)

$\mathrm{T} 2 \mathrm{c} \rightarrow 31 / 179$ (17\%)

T3a $\rightarrow$ 13/179 (7\%)

$\mathrm{T} 3 \mathrm{~b} \rightarrow 1 / 179(1 \%)$

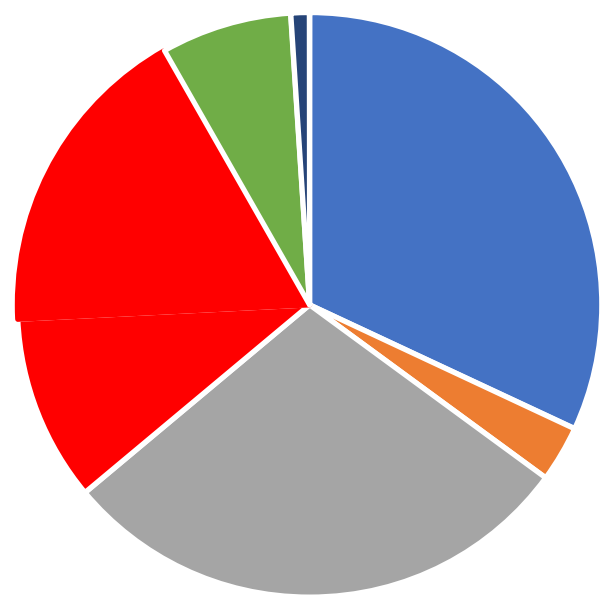

$\because \mathrm{T} 0$ - Tis $\square \mathrm{T} 2 \mathrm{a} \backsim \mathrm{T} 2 \mathrm{~b} \square \mathrm{T} 2 \mathrm{c} \backsim \mathrm{T} 3 \mathrm{a} \backsim \mathrm{T} 3 \mathrm{~b}$

Fig. 5 Graph shows the advantage of performing target + systematic sampling being able to obtain a T2b and T2c (red zone) clinical staging to better plan the surgical strategy

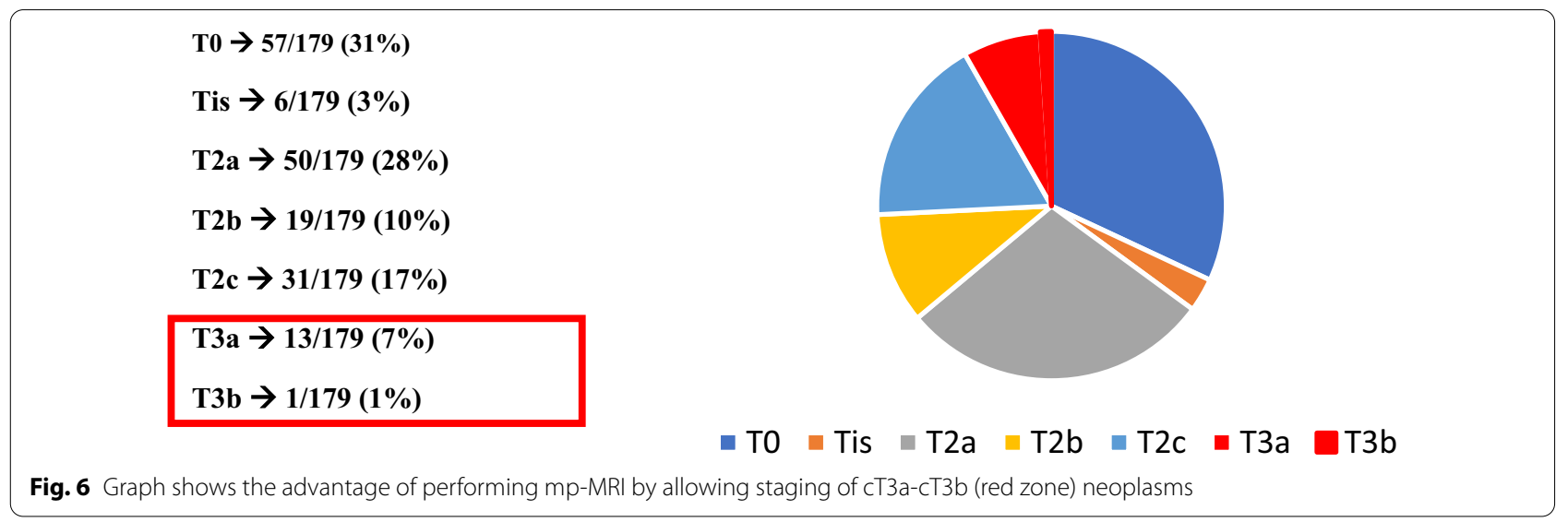

literature, as in the study by Yasuhiro Yamada et al., there is evidence that software-guided magnetic resonance imaging-ultrasound fusion-targeted biopsies offers a greater detection rate for smaller targeted lesions and also superior ability to sample greater cancer core length compared with cognitive fusion-targeted biopsies [17].

MRI provides useful information on the extent of the disease (e.g., cT3a/b) which allows you to better plan the surgical strategy or other therapies (Fig. 6).

There are limitations to the study, such as a small number of patients, the retrospectivity of the study and the degree of variability due to the execution (operator dependent) of the MRI fusion biopsy, but which can still offer interesting future outlook. In our study we used the best currently available (mpMRI in naive patients, software-based fusion biopsy) combining with a team radiological approach, obtaining a better DR, which is the main objective of our study, that is to avoid having to undergo as much as possible at rebiopsy (Table 1).

\section{Conclusion}

MRI in the future could become the gold standard for performing MRI fusion-guided biopsies to have a better diagnostic result and avoid rebiopsies. A team MRI reading allows greater accuracy in identifying the suspected lesion, which is demonstrated by a high rate of agreement with the positivity of the cores $(90.8 \%)$.

The training of the urologist who performs the biopsy and the accuracy of the MRI are fundamental for the DR of prostate cancer through MRI fusion-guided biopsy, 
Table 1 Relative Detection Rate for PIRADS score

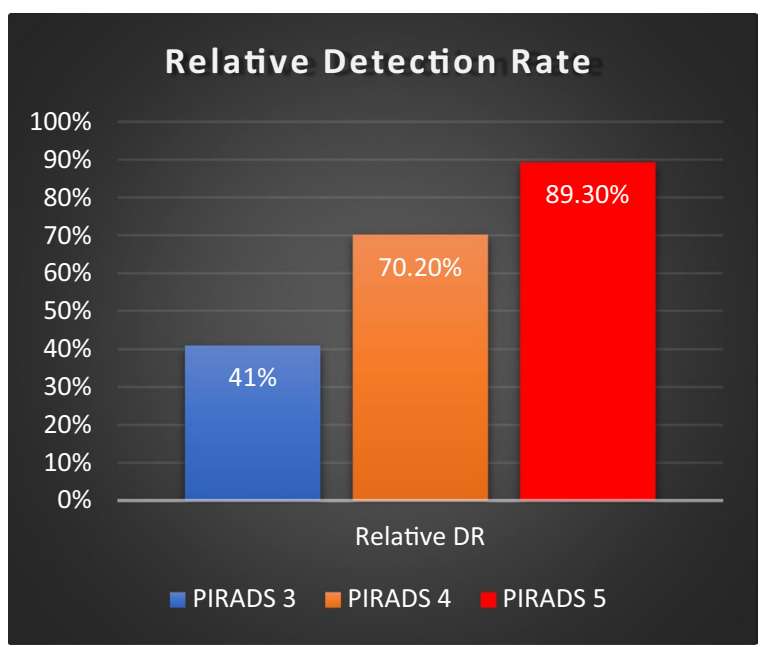

which in our experience has produced good results for the value of the MRI assessed by a multiteam of radiologists.

There is a cost problem due to the need to carry out the mpMRI but it could have less impact in the future, for example with a greater and more effective use of bPMRI or cost reduction in the procedure.

In addition, the MRI provides useful information on the extent of the disease (e.g., cT3a/b) which allows you to better plan the surgical strategy or other therapies.

\begin{abstract}
Abbreviations
MRI: Magnetic resonance imaging; TRUS: Trans-rectal ultrasound; mp-MRI: Multi-parametric magnetic resonance imaging; bp-MRI: Bi-parametric magnetic resonance imaging; DR: Detection rate; Dw: Diffusion-weighted; DCE: Dynamic contrast-enhanced; MRS: Magnetic resonance spectroscopy; DRE: Digital rectal examination; TRB: The transrectal biopsy; TPB: Transperineal biopsy; PZ: Peripheral zone; TZ: Transitional zone.
\end{abstract}

\section{Acknowledgements}

We thank our center, Versilia Hospital, for giving us the opportunity to collect the data and submit our research

\section{Authors' contributions}

All authors contributed to the creation of the article and approve its content.

\section{Funding}

We declare that we have not received funds.

\section{Availability of data and materials}

We have created the database of all patients that will be available to the journal if the article is accepted.

\section{Declarations}

\section{Ethics approval and consent to participate}

We declare that we have respected the ethical rules for data acquisition and that we have obtained the consent of the patients involved. Being a retrospective work and not being a clinical trial, we have obtained consent to use the data without having recourse to the ethics committee.

\section{Consent for publication}

We declare our consent to the publication.

\section{Competing interests}

The authors declare that they have no competing interests.

\author{
Author details \\ ${ }^{1}$ UOC Urologia Ospedale Versilia, Camaiore, LU, Italy. ${ }^{2}$ UOC Radiologia \\ Ospedale Versilia, Camaiore, Italy.
}

Received: 12 August 2021 Accepted: 8 November 2021

Published online: 04 January 2022

\section{References}

1. Ferlay J, Soerjomataram I, Dikshit R, Eser S, Mathers C, Rebelo M et al (2015) Cancer incidence and mortality worldwide: sources, methods and major patterns in GLOBOCAN 2012. Int J Cancer 136(5):E359-E386

2. Mohamed H, Sherif MM, Ragheb AM, Lotfi AM, Mikhail Ml, Ali RS et al (2019) PCN487 Cost of prostate cancer and quality of life in Egypy-a patient perspective study. Value Health 22:S531-S532

3. Kirkham APS, Emberton M, Allen C (2006) How good is MRI at detecting and characterising cancer within the prostate? Eur Urol 50(6):1163-1175

4. Riches SF, Payne GS, Morgan VA, Sandhu S, Fisher C, Germuska M et al (2009) MRI in the detection of prostate cancer: combined apparent diffusion coefficient, metabolite ratio, and vascular parameters. Am J Roentgenol 193(6):1583-1591

5. Barentsz JO, Richenberg J, Clements R, Choyke P, Verma S, Villeirs G et al (2012) ESUR prostate MR guidelines 2012. Eur Radiol 22(4):746-757

6. Barentsz JO, Weinreb JC, Verma S, Thoeny HC, Tempany CM, Shtern F et al (2016) Synopsis of the PI-RADS v2 guidelines for multiparametric prostate magnetic resonance imaging and recommendations for use. Eur Urol 69(1):41-49

7. Purnell SD, Bloom JB, Valera V, Wood BJ, Turkbey B, Pinto PA (2018) Targeted biopsy: benefits and limitations. Curr Opin Urol 28(2):219-226

8. Lourenço M, Pissarra P, Brito DV, Eliseu M, Peralta JP, Figueiredo A et al (2020) Lesion location agreement between prostatic multiparametric magnetic resonance, cognitive fusion biopsy and radical prostatectomy piece. Arch Ital Urol Androl 91(4):218-223

9. Stanzione A, Creta M, Imbriaco M, La Rocca R, Capece M, Esposito F et al (2020) Attitudes and perceptions towards multiparametric magnetic resonance imaging of the prostate: a national survey among Italian urologists. Arch Ital Urol Androl 92(4). https://www.pagepressjournals.org/ index.php/aiua/article/view/aiua.2020.4.291

10. Stabile A, Giganti F, Rosenkrantz AB, Taneja SS, Villeirs G, Gill IS et al (2020) Multiparametric MRI for prostate cancer diagnosis: current status and future directions. Nat Rev Urol 17(1):41-61

11. Kasivisvanathan V, Rannikko AS, Borghi M, Panebianco V, Mynderse LA, Vaarala MH et al (2018) MRI-targeted or standard biopsy for prostatecancer diagnosis. N Engl J Med. https://doi.org/10.1056/NEJMoa1801993

12. Tang Y, Liu Z, Tang L, Zhang R, Lu Y, Liang J et al (2018) Significance of MRI/transrectal ultrasound fusion three-dimensional model-guided, targeted biopsy based on transrectal ultrasound-guided systematic biopsy in prostate cancer detection: a systematic review and meta-analysis. UIN 100(1):57-65

13. D'Agostino D, Mineo Bianchi F, Romagnoli D, Corsi P, Giampaoli M, Schiavina R et al (2019) Comparison between "In-bore" MRI guided prostate biopsy and standard ultrasound guided biopsy in the patient with suspicious prostate cancer: preliminary results. Arch Ital Urol Androl 91(2). https://pagepressjournals.org/index.php/aiua/article/view/aiua.2019.2.87

14 Grummet J, Pepdjonovic L, Huang S, Anderson E, Hadaschik B (2017) Transperineal vs. transrectal biopsy in MRI targeting. Transl Androl Urol. 6(3):368-375

15. Gatti M, Faletti R, Calleris G, Giglio J, Berzovini C, Gentile F et al (2019) Prostate cancer detection with biparametric magnetic resonance imaging (bpMRI) by readers with different experience: performance and comparison with multiparametric (mpMRI). Abdom Radiol 44(5):1883-1893

16. Alabousi M, Salameh J-P, Gusenbauer K, Samoilov L, Jafri A, Yu H et al (2019) Biparametric vs multiparametric prostate magnetic resonance imaging for the detection of prostate cancer in treatment-naïve patients: 
a diagnostic test accuracy systematic review and meta-analysis. BJU Int 124(2):209-220

17. Yamada Y, Shiraishi T, Ueno A, Ueda T, Fujihara A, Naitoh Y et al (2020) Magnetic resonance imaging-guided targeted prostate biopsy: comparison between computer-software-based fusion versus cognitive fusion technique in biopsy-naïve patients. Int J Urol 27(1):67-71

\section{Publisher's Note}

Springer Nature remains neutral with regard to jurisdictional claims in published maps and institutional affiliations.

\section{Submit your manuscript to a SpringerOpen ${ }^{\circ}$ journal and benefit from:}

- Convenient online submission

- Rigorous peer review

- Open access: articles freely available online

- High visibility within the field

- Retaining the copyright to your article

Submit your next manuscript at $\boldsymbol{\nabla}$ springeropen.com 